$16^{\text {th }}$ International Conference on

AEROSPACE SCIENCES \& AVIATION TECHNOLOGY,

ASAT - 16 - May 26 - 28, 2015, E-Mail: asat@ mtc.edu.eg

Military Technical College, Kobry Elkobbah, Cairo, Egypt

Tel : +(202) 24025292 - 24036138, Fax: +(202) 22621908

\title{
Space Operations Monitoring Based on a New Statistical Principle Component Analysis Algorithm
}

\author{
B. Nassar*, W. Hussein ${ }^{\dagger}$ and M. Mokhtar*
}

\begin{abstract}
The crucial concern of satellite operations is to ensure the health and safety of satellites. The worst case in this perspective is probably the loss of a mission but the more common interruption of satellite functionality can result in compromised mission objectives. All the data acquiring from the spacecraft are known as telemetry (TM), which contains the wealth information related to the health of all its subsystems. Each single item of information is contained in a telemetry parameter, which represents a time-variant property (i.e. a status or a measurement) to be checked. As a consequence, there is a continuous improvement of TM monitoring systems in order to reduce the time required to respond to changes in a satellite's state of health. A fast conception of the current state of the satellite is thus very important in order to respond to occurring failures. Statistical multivariate latent techniques are one of the vital statistical learning tools that are used to tackle the aforementioned problem coherently. Information extraction from such rich data sources using advanced statistical methodologies is a challenging task due to the massive volume of data. To solve this problem, in this paper, we present a new unsupervised statistical algorithm based on principle component analysis (PCA) latent technique. The algorithm is particularly applied to an actual remote sensing spacecraft. Data from the attitude determination and control subsystem (ADCS) was acquired under two operation conditions: normal and faulty states. The models were built and validated under these conditions and the results shows that the algorithm could successfully differentiate between these operations conditions. Furthermore, the algorithm provides competent information in prediction as well as adding more insight and physical interpretation to the ADCS operation.
\end{abstract}

Keywords: System Monitoring; Multivariate Analysis; PCA Algorithm; Space Operations.

\footnotetext{
*Egyptian Armed Forces, Egypt, bassemamo@yahoo.com

${ }^{\dagger}$ Egyptian Armed Forces, Egypt, wessam.hussein@gmail.com

"Professor of Electrical Engineering and Chairman of the National Authority for Remote Sensing and Space Sciences m.medhat@narss.sci.eg
} 


\section{Introduction}

Reliability, availability, and safety are critical requirements in space mission operations. Moreover flight controllers' are responsible not only for operating their designated spacecraft subsystems to meet mission intentions, but also for monitoring those subsystems to ensure that they are operating appropriately.

Data-driven process monitoring or statistical process monitoring (SPM) applies multivariate statistics and machine learning methods to fault detection and diagnosis for space operations, which has become one of the most rich areas in research and practice over the last two decades. Based on methods from multivariate statistical analysis, SPM has found wide applications in various processes, including space operations. The situation is greatly complicated by the fact most of the techniques representing different modalities are complex and have a black box impacts. So, due to the data-based nature of the SPM methods, it is relatively easy to apply to real processes of rather large scale comparing to other methods based on systems theory or rigorous process models.

Space missions are characterized by large-scale complex operations that are well instrumented with a multi-level control hierarchy, making it a suitable place to apply the SPM methodologies. Disturbances and variabilities in operation conditions are the critical obstacles to overcome in order to make quality operations. The task of data-driven process monitoring is to detect such an abnormal situation and diagnose the root-cause early. SPM capabilities are to extract vital information from both huge archived and real time operations data. The Applications of SPM methodology provides better comprehension and more accurate characterization of changes and disturbances in space systems operations.

The paper represents an unsupervised statistical fault detection algorithm based on principle component analysis (PCA) latent space methodology. The algorithm introduces a practical approach for monitoring and diagnosis that includes: (i) fault detection; (ii) fault diagnosis and quality monitoring. The tasks of the algorithm are to model, analyze and identify key contributors to anomalous events automatically which lead to characterize the attitude determination and control subsystem (ADCS) behavior of the spacecraft. However, this approach doesn't build a causality direction between variables rather it builds a correlation direction inside a bounded region. Hence, it represents an efficient tool as a first step for process monitoring through the analysis of existing information. The contributions of this design work can be itemized as:

1. To the best of the authors' knowledge, this is a new application to model and analyze the ADCS spacecraft telemetry using the unsupervised statistical learning algorithm PCA.

2. The algorithm possesses the modelling of the data in the X-space where the advantage of the contribution and control plots can be seen. These plots facilitate the detection of the variables responsible for any process and monitor key process variables and quality over time.

The remainder of the paper is organized as follows. We introduce an overview of related work to the area of research in section 2. The proper use of data-driven models based on multivariate latent space philosophy is discussed in section 3. The ADCS configuration is presented in section 4. Then, the main details and theoretical analysis of the algorithm are discussed in section 5. The performance of the algorithm on the ADCS telemetry data and the analysis results are compared with multivariate analysis software SIMCA-P developed by Umetrics are shown in section 6 and 7. Finally, we conclude and discuss about future in section 8 . 


\section{RELATED WORK}

Archived telemetry contain a wealth of information about the complex space system behavior, so recent development techniques for monitoring and anomaly detection make it possible to examine this archived data and extract embedded information to produce advanced system health monitoring applications [1]. Researchers have devoted considerable effort to the application of various different soft computing methods to develop heath monitoring systems (HMSs) for space operations. The methods used include neural networks, multivariate statistical approaches and data mining techniques. These methods can provide important tools for the field of intelligent monitoring which can learn, adapt, and make decisions concerning the system they are in charge of [2].

Takehisa Yairi et al. [3] evaluate a variety of dimensionality reduction algorithms and compare between them without using the cross-validation. Regarding to the authors point of view using the cross-validation might be too time-consuming when the training set or the number of classes is large.

Ivano Verzola et al. [4] clarify that a space operations are often based on a reactive model. The main drawback of this model is the impossibility of preventing the failure executing preventive actions to avoid the expected faulty condition. Moreover, their work describes a study on a possible proactive model to deal with failures based on techniques from statistics, machine learning and data mining to identify future trends of the object to foresee the behavior of the system. But, the research work didn't give the predictions in real-time that to be checked against a set of pre-defined failure probabilities thresholds.

John MacGregor et al. [5] establish the potential of applying multivariate methods in monitoring and fault diagnosis contrasted with many other data-driven techniques. The authors declared that black-box models such as artificial neural networks (ANNs) and hidden markov models (HMM) and statistical classification techniques such as discriminant analysis (DA) and support vector machines (SVMs) are fallen within the class of regression methods/classifiers that provides no allowance for modeling the X-space. Also, those techniques have a limited capability to interpret of full rank data, to handle missing data and to test for outliers in new data. Even though they recognize that methods can be useful in some cases.

Jian Peng et al. [6] propose a fault diagnosis method for key components of satellite called anomaly monitoring method (AMM), which is made up of state estimation based on multivariate state estimation techniques (MSET). This method applied to the satellite power subsystem and the analysis of failure applied on lithium-ion batteries (LIBs). Only two parameters were selected as the key parameters of AMM, so either an in-depth analysis failure of LIBs be conducted or more influencing parameters were considered. Steve Lindsay et al. [7] apply several supervised learning and unsupervised methods to four different spacecraft state-of-health $(\mathrm{SOH})$ scenarios after the data pre-processing step. But real-time anomaly resolution still remains a potential research area to be examined.

The simplicity of multivariate statistical analysis approach is that there is no need for a fundamental model of the system and only data from normal operation needs to be used, which is generally available in some form for most systems. Among the approaches used in multivariate analysis there are two projection methods called principal component analysis (PCA) and projection to latent structure (PLS). Many applications of these two methods have been successfully applied in the fields of process monitoring. Among these techniques used in multivariate analysis, the PCA is a well-known unsupervised multivariate statistical tool used in many applications particularly the fields of process monitoring and diagnosis [8, 9-10]. 


\section{Multivariate PCA Basic Theory}

The principle component analysis (PCA) is a quantitatively rigorous unsupervised statistical learning technique that used in extracting information from data and it is widely used by scientists and engineers in various disciplines such as in process monitoring, data compression, image analysis, as well as in fault detection decades ago. The PCA methodology is based on projection philosophy that aims to find a line, a plane or hyperplane "latent direction" which maximize the variance of the projection. So, the PCA is a transformation or mapping of the correlated variables from its original space to a lower dimensional space "new variables" that are uncorrelated with each other and are linear combinations of the original variables and also preserving the correlation structure between the original features. The method produces a compressed statistical model that gives linear combinations of the original variables which describe the major trends in a datasets in terms of capturing the variance of the data. The "aggregate variance" of the whole set of variables remains unchanged from before to after the mapping, but the variance is redistributed so that the most is in the first mapped variable, the next largest amount goes to the second mapped variable, and the least to the last transformed variable. These new variables are called the principal components [13]. The utility of this method lies in the ability to explain as much of the total variation in the data as possible with the least number of principal components "new variables".

Fig. 1. illustrates the projection philosophy by an imaginary example measuring three different variables, $\mathrm{X} 1, \mathrm{X} 2$, and $\mathrm{X} 3$ (part A). First we scale the data by subtracting the mean and dividing by the standard deviation. Thus, the centroid of the whole data set is zero (part B). We label these scaled axes S1, S2, and S3 with the relative location of the points remaining the same. We can notice a clear slope from the lower left front to the upper right back. By drawing a line along this slope the first PCA axis is built as that line that goes through the centroid but also minimizes the square of the distance of each point to that line. Thus, the line is as close to all of the data as possible (part C). Equivalently, the line goes through the maximum variation in the data. The second PCA axis also must go through the centroid, and also goes through the maximum variation in the data, but with a certain constraint that it must be completely uncorrelated (i.e. orthogonal) to PCA axis1 (part C). If we rotate the coordinate frame of PCA axis1 to be on the X-axis, and PCA axis2 to be on the Y-axis, then the last figure (part D) is obtained. Every axis has an eigenvalue (latent root) associated with it, and they are ranked from the highest to the lowest. These are related to the amount of variation explained by the axis [8]. 

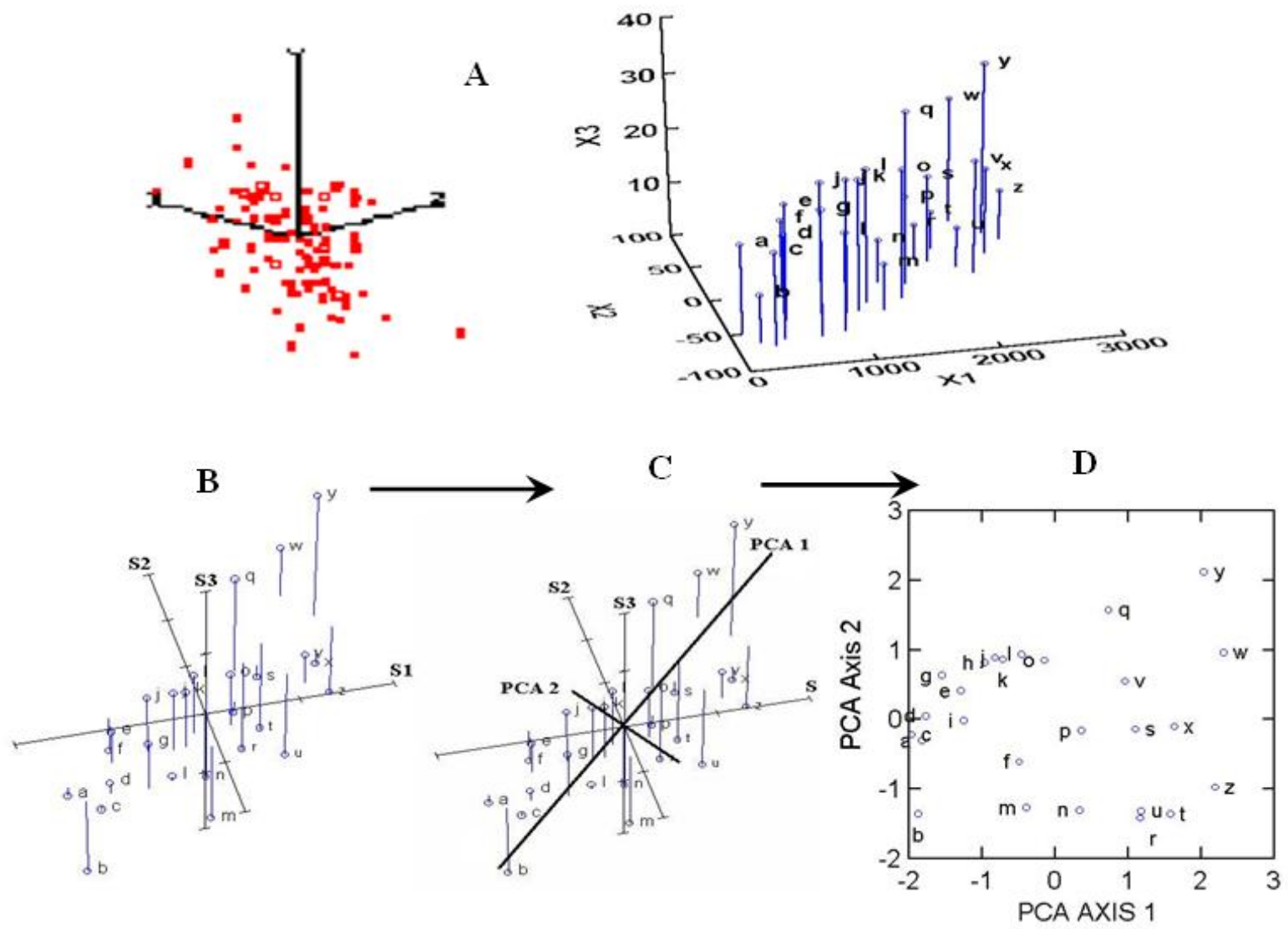

Fig. 1. Projection Philosophy of Multivariate Techniques

The point that needs to be stressed is that the data can be reduced to a size which is more manageable but contains the features that are often of interest. The PCA extracts a score matrix, $\mathrm{T}$, and a loading matrix, $\mathrm{P}$, from $\mathrm{X}$. These matrices have the following dimensions $[9,11]:$

$X: N \times K \quad T: N \times A \quad P: K \times A$

The first column of $\mathrm{T}$ and $\mathrm{P}$ are called by their shorter forms, $\mathrm{t} 1$ and $\mathrm{p} 1$ respectively. For a given data matrix-X, which has (m)i rows of samples and (n) columns of variables, each row $\mathrm{X}_{\mathrm{i}}^{\mathrm{T}}$ is a sample of $\mathrm{n}$ variables associated with a given time. In this study, the data matrix contains values of several variables from spacecraft telemetry during normal process operation, a time when the operation was running in a desirable state. If the columns of $\mathrm{X}$ are mean centered, the covariance matrix of $\mathrm{X}$ is:

$\operatorname{cov}(\mathrm{X})=\left(\frac{1}{m-1}\right)\left(X^{T} X\right)$

Columns of $\mathrm{X}$ are mean centered by subtracting the mean of each column from each entry in that column. If $\mathrm{X}$ has been autoscaled (mean centered and adjusted to the unit variance by dividing each column by its standard deviation), the covariance matrix is the correlation matrix PCA decomposes "decomposition by variance" the data matrix-X to the sum of the products of $K(K \leq \min \{m, n\})$ pairs of vectors and a residual matrix, $E$ :

$\mathrm{X}=\mathrm{t}_{1} p_{1}^{T}+\mathrm{t}_{2} p_{2}^{T}+\ldots \ldots+\mathrm{t}_{\mathrm{k}} p_{k}^{T}+\mathrm{E}$

$\mathrm{t}_{\mathrm{i}}=$ scores vectors

$\mathrm{P}_{\mathrm{i}}=$ loadings vectors (eigenvectors of the covariance matrix)

$\mathrm{E}=$ residual matrix 
So, the score vectors in the score plot represent the principle component $\left(t_{i}\right)$ "new variables" and $\left(\mathrm{p}_{\mathrm{i}}\right)$ the loadings vectors represent (eigenvectors of the covariance matrix) or variables coefficients. In addition, the score and loading plots are complementary and superimposed, this means that variables lying in each quarter of the loading plot are contributing to the changes in the observations in the score plot. The residual matrix contains that part of the data not explained by the PCA model. The N-K t, $\mathrm{p}$ vector pairs not in the model are associated with "noise" the uncontrolled process and or instrument variation arising from random influences. The scores vectors $\left(t_{i}\right)$ form an orthogonal set $\left(t_{i}^{T} t_{j}=0\right.$ for $\left.i \neq j\right)$ and describe how the samples relate to each other. The loadings vectors $\left(\mathrm{p}_{\mathrm{i}}\right)$ are orthonormal $\left(p_{i}^{T} p_{\mathrm{j}}=0\right.$ for $\mathrm{i} \neq \mathrm{j}$, $p_{i}^{T} \mathrm{p}_{\mathrm{j}}=1$ for $\mathrm{i}=\mathrm{j}$ ) and describe how the variables relate to each other. These variables $\left(\mathrm{p}_{\mathrm{i}}\right)$ are the eigenvectors of the covariance matrix and $\left(\lambda_{\mathrm{i}}\right)$ is the eigenvalue corresponding to the eigenvector $\left(\mathrm{p}_{\mathrm{i}}\right)$, subsequently the covariance matrix equal to $\operatorname{cov}(X) \mathrm{p}_{\mathrm{i}}=\lambda_{\mathrm{i}} \mathrm{p}_{\mathrm{i}}$. A more indepth discussion, which also highlights some geometric concepts of PCA, can be found in [9]. The algorithm used to calculate the PCA is the nonlinear iterative partial least squares algorithm (NIPALS) [9, 11].

\section{System Configuration}

Actually, the spacecraft telemetry data contains a variety of information including myriad sensor measurements. Anomaly detection techniques described in the previous section was applied to the attitude determination and control system (ADCS) of remote sensing spacecraft owned by National Authority for Remote Sensing and Space Sciences (NARSS).The main purpose of the ADCS is to orientate the main structure of the spacecraft at the desired angle(s) within required accuracy. Attitude of spacecraft can be represented in different ways with set of variables such as directions cosine matrix (DCM), Euler angles, angular velocity and quaternion also known as "Euler parameters"...etc. The ADCS composition contains angular velocity meter (AVM) blocks which are mounted in such a way that three AVMs measuring axes are collinear to body-fixed axes, and the fourth one is a backup. The system also had a magnetometer (MM), magnetorquers (MT), reaction wheels (RW) and star sensor. Fig. 2. shows the space system structure.

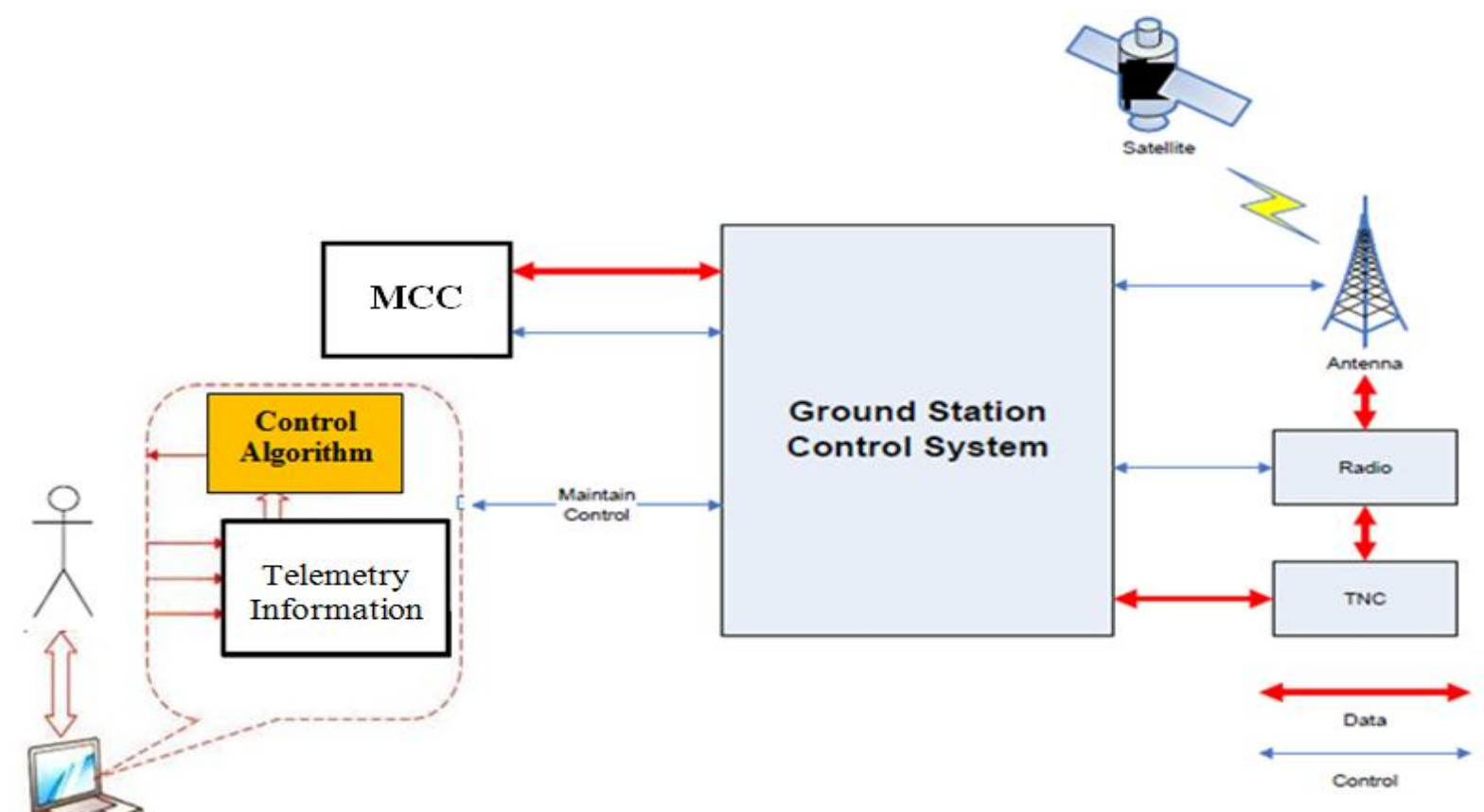

Fig. 2. Structure Diagram of the Space System 


\section{Algorithm Peculiarities}

The principle component analysis (PCA) algorithm is mainly applied as an unsupervised statistical learning tool to the spacecraft telemetry. The algorithm strategy is to manage operation status acquired via the attitude determination and control subsystem (ADCS) telemetry data to provide efficient monitoring for the aforementioned system. The methodology operates in two phases; first the training phase learns the model using the available training data. Then, the next phase trends and clusters the data instance as normal or an outlier using the model. By outliers here we mean both strong and moderate outliers. Strong outliers mean strong deviations in the systematic part of the data and they have leverage "influence power" on the model, because they can shift the model plane towards themselves. Leverage measures the influence of an observation and it proportional to the distance of observation from the center of the data. While by moderate outliers we mean outliers that aren't strong enough to shift the model plane but they provide an early indication to process upset. The framework of the algorithm enumerated as follows:

1. Collect the ADCS telemetry from sensory orbit of the normal operation as the training data.

2. Auto-scaling "standardization" the training data to the unit variance and mean centering "variance $=1$ and mean $=0$ " to unify the influence of the features before building the model. These measures allow the model to decide the affect of each variable regarding their real influence.

3. Train the PCA model using the training data (Training samples $X=\left[X_{1}, X_{2}, . ., X_{m}\right]$. Then, calculate the variance of matrix $(X)$ which measure of the spread of data in a given data set (one dimensional concept):

$\operatorname{Var}(\mathrm{X})=\frac{\sum_{\mathrm{i}=1}^{\mathrm{m}}\left(\mathrm{X}_{\mathrm{i}}-\overline{\mathrm{X}}\right)\left(\mathrm{X}_{\mathrm{i}}-\overline{\mathrm{X}}\right)}{\mathrm{m}-1}$

Where, $\bar{X}$ mean of the set $X$ and $\mathrm{m}$ : sample number and $\Lambda=\operatorname{diag}\left(\lambda_{1}>\lambda_{2}>\ldots .>\lambda_{\mathrm{m}}\right)$ is a diagonal matrix containing the eigenvalues in a decreasing order $\left(\lambda_{1}>\lambda_{2}>\ldots .>\lambda_{m}\right)$. Further, to calculate the covariance matrix (S) of data Matrix (X), so if the data matrix$\mathrm{X}$ has $\mathrm{m}$-rows and $\mathrm{n}$ columns, then the covariance matrix $\mathrm{S}$ is equal to:

$\mathrm{S}=\operatorname{cov}(\mathrm{X})=\left(\frac{1}{m-1}\right)\left(X^{T} X\right)$

Subsequently, choose how many PCs used.The goodness of the PCA model depends on a good choice of how many principal components (PCs) are retained. Underestimating the number of PCs can leave out important variations in the data which degrades the prediction quality of the PCA model. Overestimating the number of PCs, on the other hand, introduces noise that masks some of the important features in the data.

4. Construct the model for exploratory and trend the data under diverse operating conditions. Then, compute the ranking of all features with a certain criterion in term of their contribution to trends and clustering. One can note that the ranking criterion for the trends and clustering of features is based on the normal vector of the hyper-plane.

5. Finally, the model is used along with one of the detection guides the Hotelling's $\left(\mathrm{T}^{2}\right)$ and the $(\mathrm{Q})$ statistic or squared prediction error (SPE) to detect faults for new data samples (if the detection index falls outside the control limits, which are defined by the thresholds associated with these indices). The Hotelling's $\left(\mathrm{T}^{2}\right)$ statistic measures the variations in the principal components at different time samples. For new testing data, when the value of $\mathrm{T}^{2}$ exceeds the value of the threshold, $\left(\mathrm{T}^{2} \alpha\right)$ a fault is declared. The (Q) statistic measures the projection of a data sample on the residual subspace, which provides an overall measure of how a data sample fits the model. When a vector of new data is available, the Q statistic is calculated and compared with the threshold value $(\mathrm{Q} \alpha)$. If the confidence limit is violated, a fault is declared. The value of threshold is 
calculated based on the assumptions that the measurements are time-independent and multivariate normally distributed. The concept of using the $\mathrm{Q}$ and $\mathrm{T}^{2}$ statistics for fault detection can be explained graphically with a simple example shown in Fig. 3. in which two faults occur. One fault (represented by the hollow circle) is detectable by both the $\mathrm{T}^{2}$ and Q statistics because it lies outside the control limit of both of them. The other fault (represented by the full circle), however, is detectable by the Q statistic, but not by the $\mathrm{T}^{2}$ statistic. This is because the $\mathrm{T}^{2}$ statistic provides a measure of the deviation in the PCs that are of greatest importance to the normal process operation. Thus, the normal operating region defined by the $\mathrm{T}^{2}$ control limits is usually larger than that defined by the $\mathrm{Q}$ control limits. Therefore, faults with small magnitudes can easily exceed the $\mathrm{Q}$ threshold, but not the $\mathrm{T}^{2}$ threshold, which makes the $\mathrm{Q}$ statistic usually more sensitive than $\mathrm{T}^{2}$ for this type of faults. This explains why the $\mathrm{Q}$ statistic is considered more preferable for fault detection than $\mathrm{T}^{2}$. A more discussion which also highlighted some mathematical concepts of $\mathrm{T}^{2}$ and $\mathrm{Q}$ statistics calculations could be found in $[15,16]$.

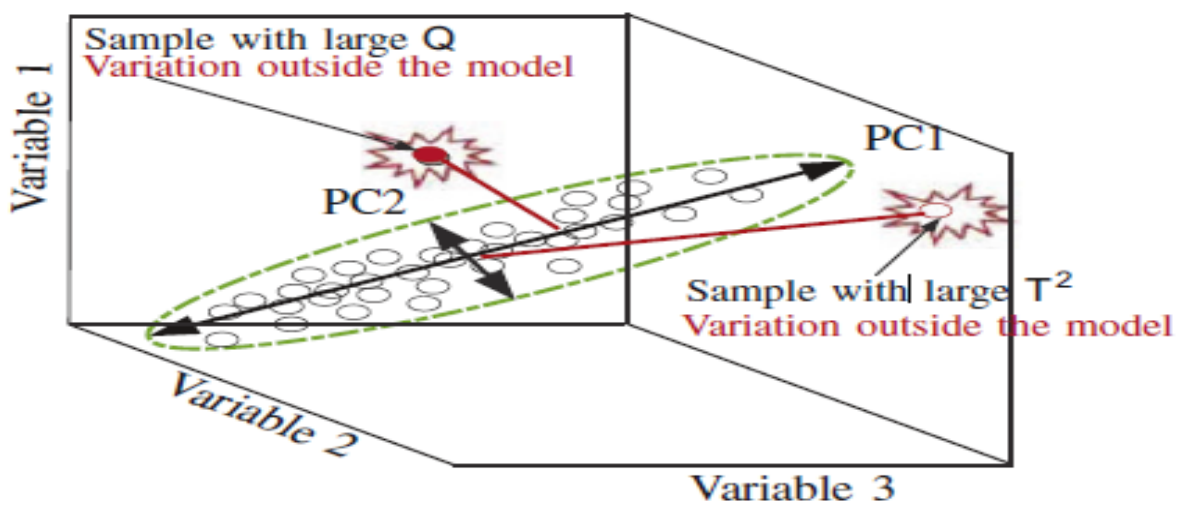

Fig. 3. Effect of Using the $T^{2}$ and $Q$ Statistics as Fault Detection Indices.

In our paper, only $\mathrm{Q}$ statistic is used as a benchmark for fault detection. Fig. 4. shows the framework of the PCA monitoring algorithm.

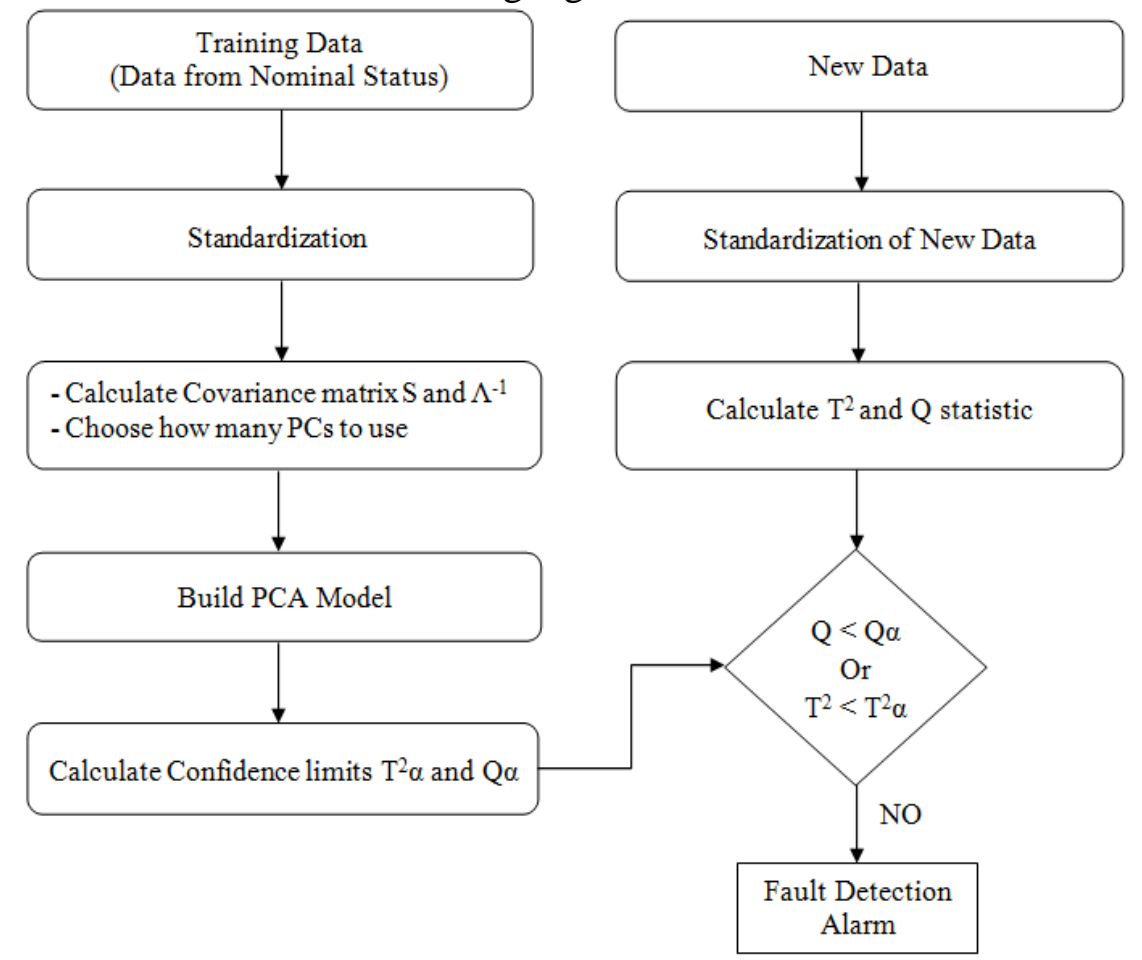

Fig. 4. PCA Fault Detection Algorithm Framework 


\section{Model Building}

The concept of the model depends on using of operation variables. Let X-matrix includes variables containing information from the sensory data. First, the data were mean centred and scaled to unit variance. Second, control limits in the latent space were established using F-distribution based on reference distribution provided by the dataset. Also, t 1 and $\mathrm{t} 2$ are the first two principal components that capture most of the variance in the X-matrix. Fig. 5. illustrates how the score plot is built for a simple case 3-variables and 2-scores. After determining the direction of maximum variation by iterative steps, one might rotate the new plane determined by the new score variables $\mathrm{t} 1$ and $\mathrm{t} 2$ and then monitor the change of the new observations in the reduced dimensional space during space operations.

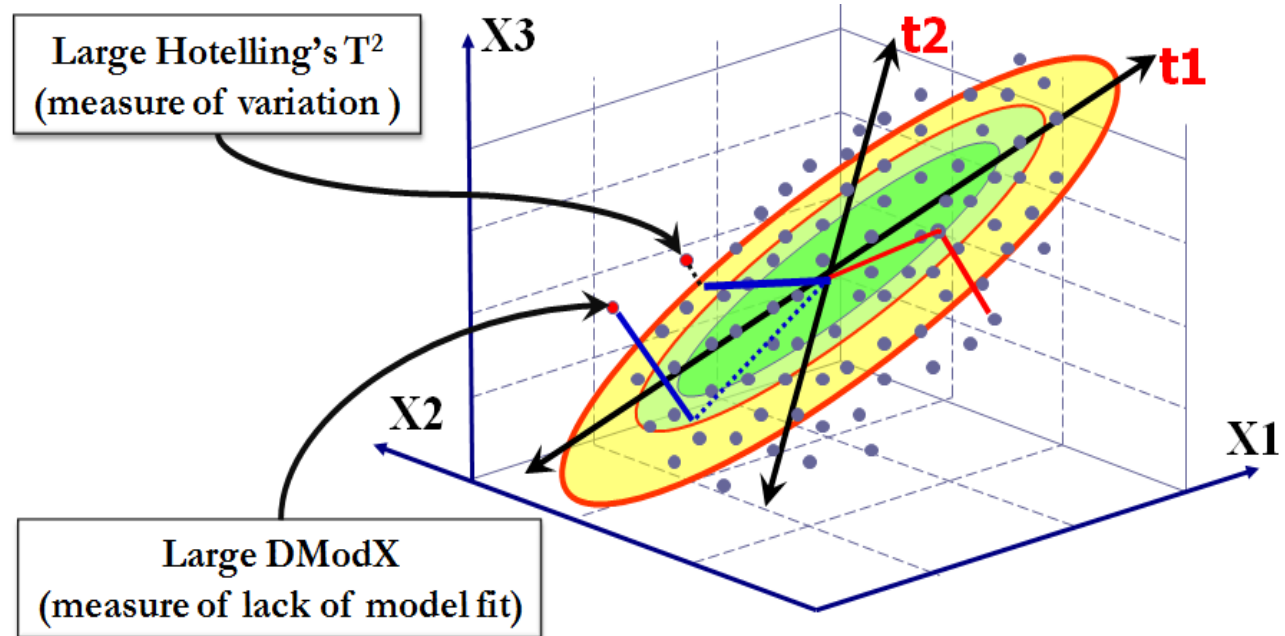

Fig. 5. Establishing Score Plot for Three Variables

The scenario of spacecraft operation uses a total of "16" features to generate the input matrix $-X$, which means that the original data has "16 dimensions means $D=16 "$. These features represent the root mean square of the time domain records of the sensory signals measurements regarding spacecraft roll, pitch, yaw angles, angler velocity and quaternion's --etc,. Models are built using the new principle component analysis (PCA) algorithm and compared with SIMCA-P software developed by Umetrics [9]. The PCA finds latent directions that maximize the variance of the projection. The multivariate model is a linear model given in matrix form by: $\mathrm{X}=\mathrm{TP}^{\mathrm{T}}+\mathrm{E}$. However, there exist non-linear PCA versions even by augmenting the original matrix with the non-linear factors or by building a non-linear relation between score factors [14]. The models are established using a set of telemetry data sampled from the spacecraft operation for two days that represents the nominal and off nominal operations of the attitude determination and control subsystem (ADCS). During this period, a system anomaly was reported due to angler velocity meter in Z-direction (AVMz) malfunctioning and the reserve one (AVMr) was used instead of faulty device. In addition spacecraft high rate damping mode (detumbling mode) occurs. If the aforementioned fault will be repeated the spacecraft will acquire the detumbling mode more than the design specifications and regarding to experts experience this mode is very critical because it could be lead to spacecraft losses.

\section{Results and Analysis}

The key target in building the principle component analysis (PCA) models is data preparation, scaling, and selecting the number of principal components. First, data must be in tab enclosed text format with no headings or extra characters before entering into MATLAB. The PCA is an unsupervised statistical learning approach which does not have class labels on training data and sometimes does not include the number of classes. The approach is based 
on using an available database of real telemetry data that gives an acceptable level of process quality to build the monitoring system and then the model can reveal both smooth time trends and sudden shifts in the telemetry of normal operation. Consequently, the first model is generated using the proposed PCA algorithm and compared with SIMCA-P software model, so a clear supervision for the data can be attained. The telemetry with "770" observations from nominal operation as training set for different variables values used to develop the PCA model. Fig. 6. shows a scatter plot of the two score vectors $t 1$ and $t 2$ of the PCA algorithm (new mapping variables). The model illustrates a clear direction of the data that indicates the trends. Fig. 7. demonstrates the results acquired by SIMCA-P software. To get better supervision of the data another attempt has been made by augmenting the $\mathrm{X}$-matrix using transformation and unit scaling of variables. The model is fitted by cross-validation to get a two component model with: R2X(cum): 0.893, Q2(cum): 0.812. The model shows a good percentage of explanation of the data $\mathrm{R} 2 \mathrm{X}(\mathrm{cum})$, together with a good percentage in the prediction ability as shown by $\mathrm{Q} 2(\mathrm{cum})$ and the both models results have a remarkable agreement.

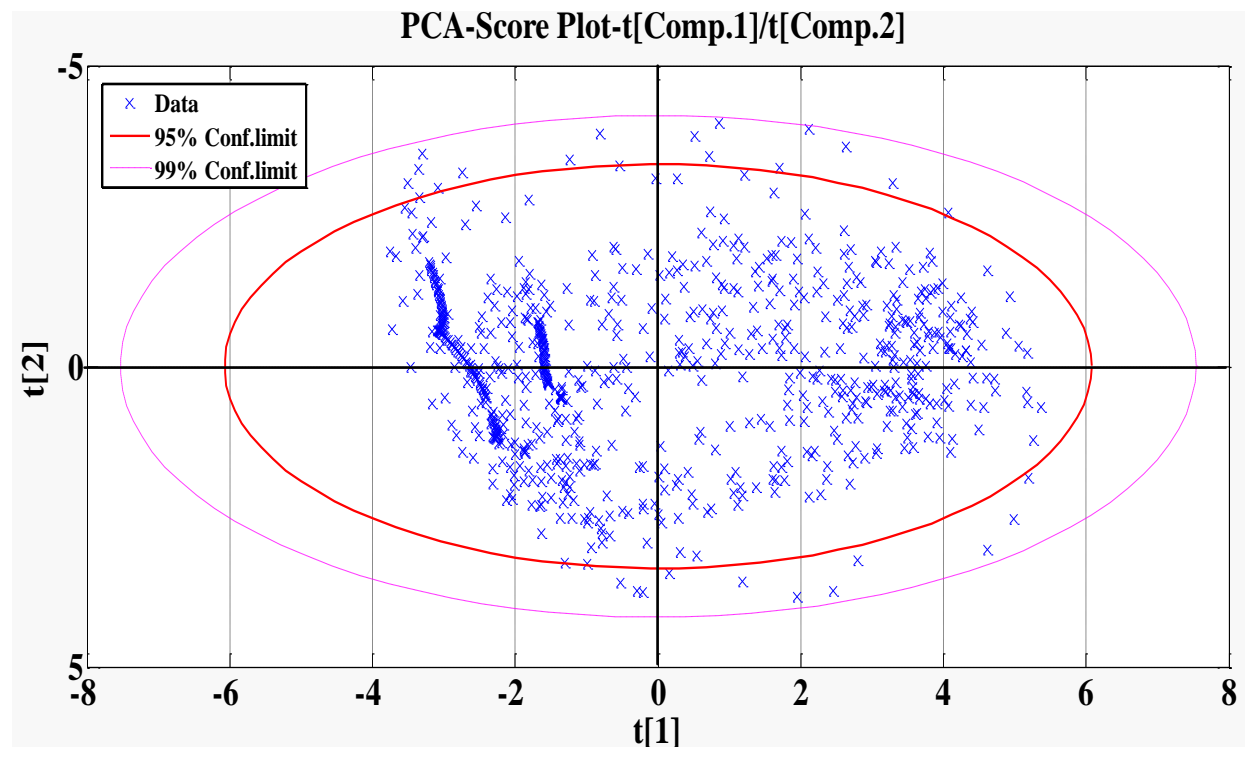

Fig. 6. PCA Score Vectors (t1 and t2) of the Algorithm using Nominal Process Data

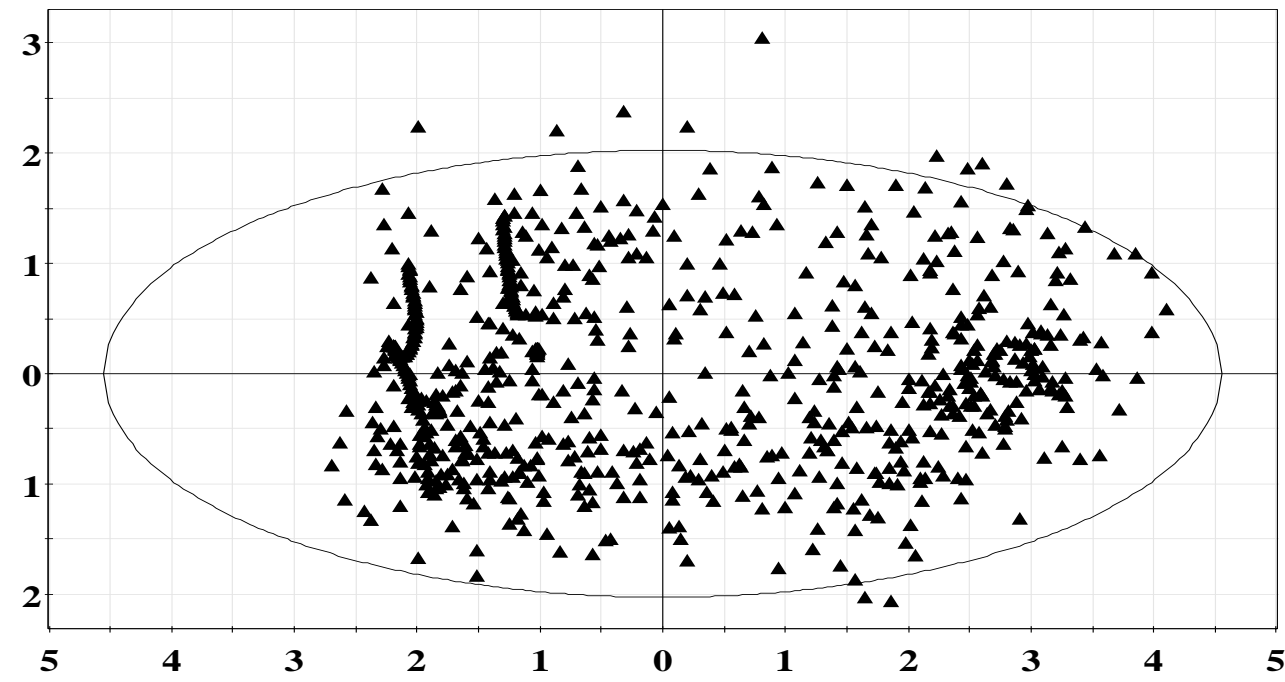

Fig. 7. PCA Score Vectors (t1 and t2) of SIMCA-P/SW using Nominal Process Data 
A model developed from a training set must be validated and tested with new observations not included in the original training set. Hence, the second PCA model was developed use a test set of the "37" observations "7 normal, 33 faulty". As we mentioned in section 3 and referred to nonlinear iterative partial least squares (NIPALS) algorithm that $\mathrm{T}=\mathrm{P}^{\mathrm{T}} \mathrm{X}$, so the score vectors in the score plot represents the principle component (new variables) ti and pi represent the loadings vectors (eigenvectors of the covariance matrix) or variables coefficients. Fig. 8. scatter and loading plots of the model. The scatter plot of the two score vectors ( $\mathrm{t} 1$ and $\mathrm{t} 2$ ) provides a clear vision of the dispersion of the data, with two main groups: normal and faulty data. This clustering is shown by manually highlighted by black line clusters in the plot (for visual purposes). The analysis proves the capability of the model to create unique data clusters and compare the characteristics of each one. Also, a clear interference can be detected between two groups in observation "N 771" and it is shown by manually highlighted with red dotted circle in the score plot and the explanation for this will be introduced afterward. Additionally, another attempt has been made by expanding the $\mathrm{X}$-matrix with square terms, cross product and cubic terms between the important variables that represent the most effective influence regarding to the fault.

More study is conducted by examining the loading plot of the model in order to investigate the relationships between the different variables. Fig. 8. shows that the loading plot clarifies the relations between variables. As we explain before the score and loading plots are complementary and superimposed, so by investigating the loading plots one can detect directly which variables are more responsible for affecting a specified group of data in the score plot.

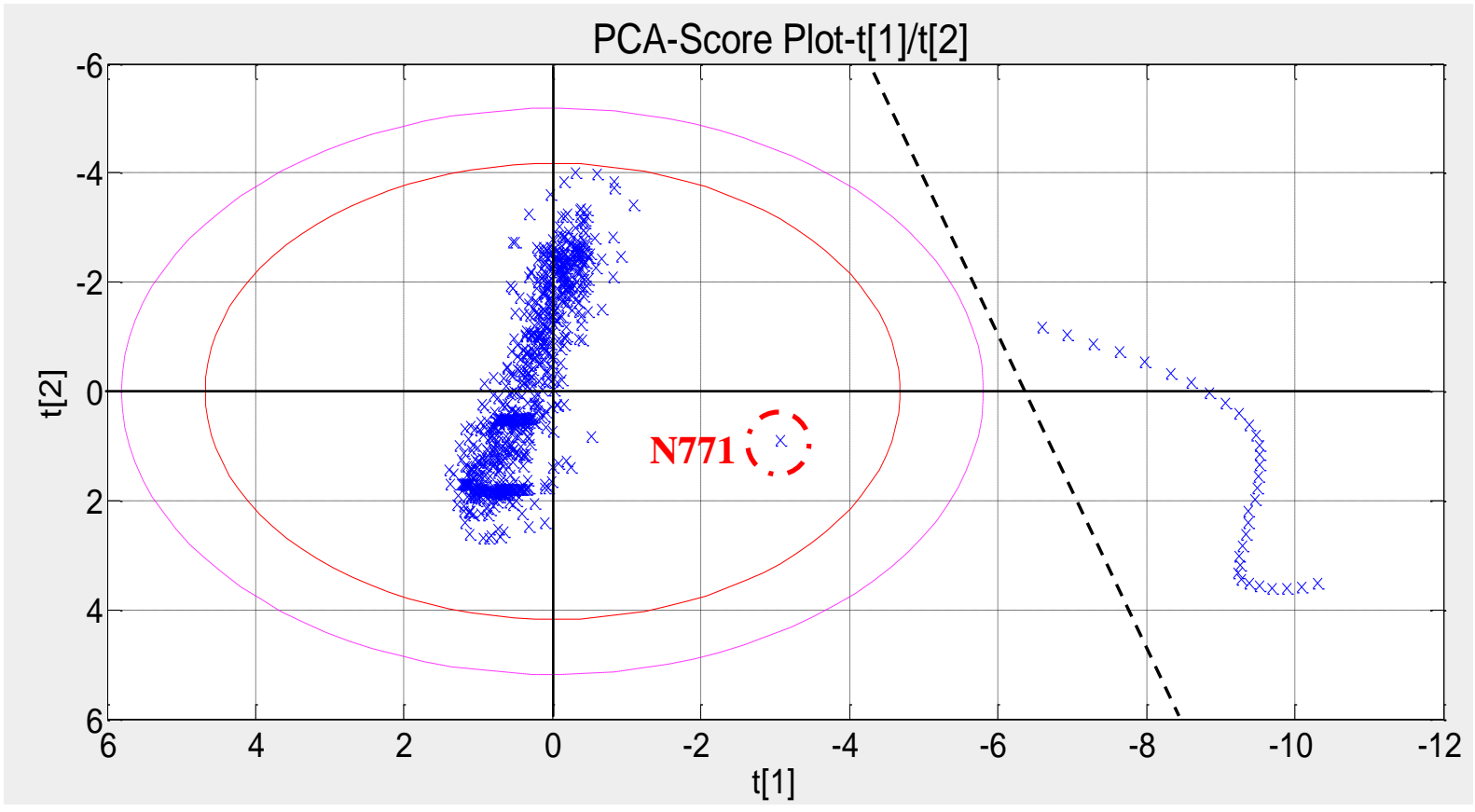




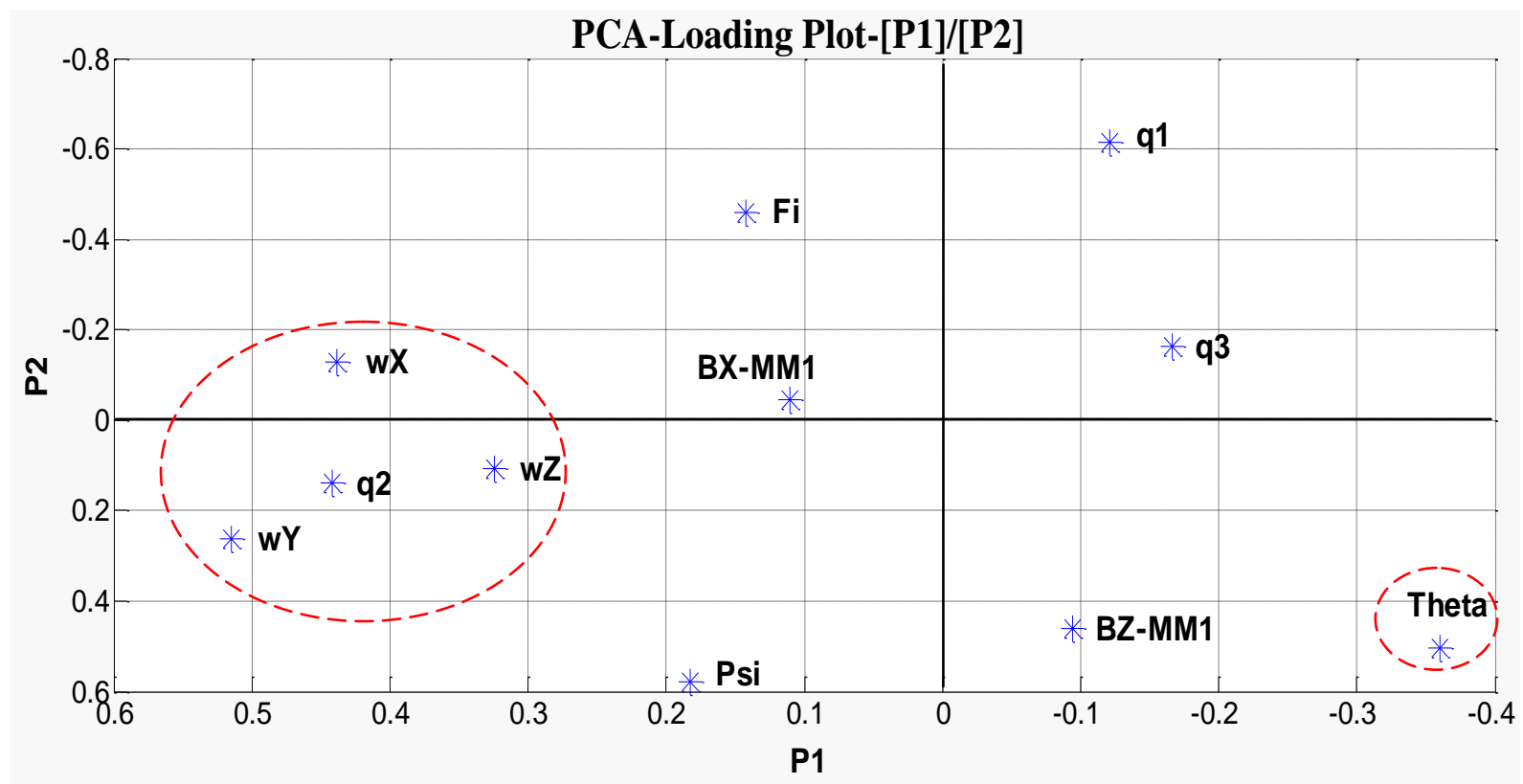

Fig. 8. PCA Algorithm Score and Loading Plots using the Training Dataset

Fig. 9. demonstrates the results acquired by SIMCA-P software, and the model is fitted by cross-validation to get a two component model with R2X(cum): 0.882, Q2(cum): 0.793. The model shows a good percentage of explanation of the data R2X(cum), together with a good percentage in the prediction ability as shown by Q2(cum) and finally, assessment between both models results shows a significant agreement.

PCA-X_t tComp. 1]/t[Comp. 2]

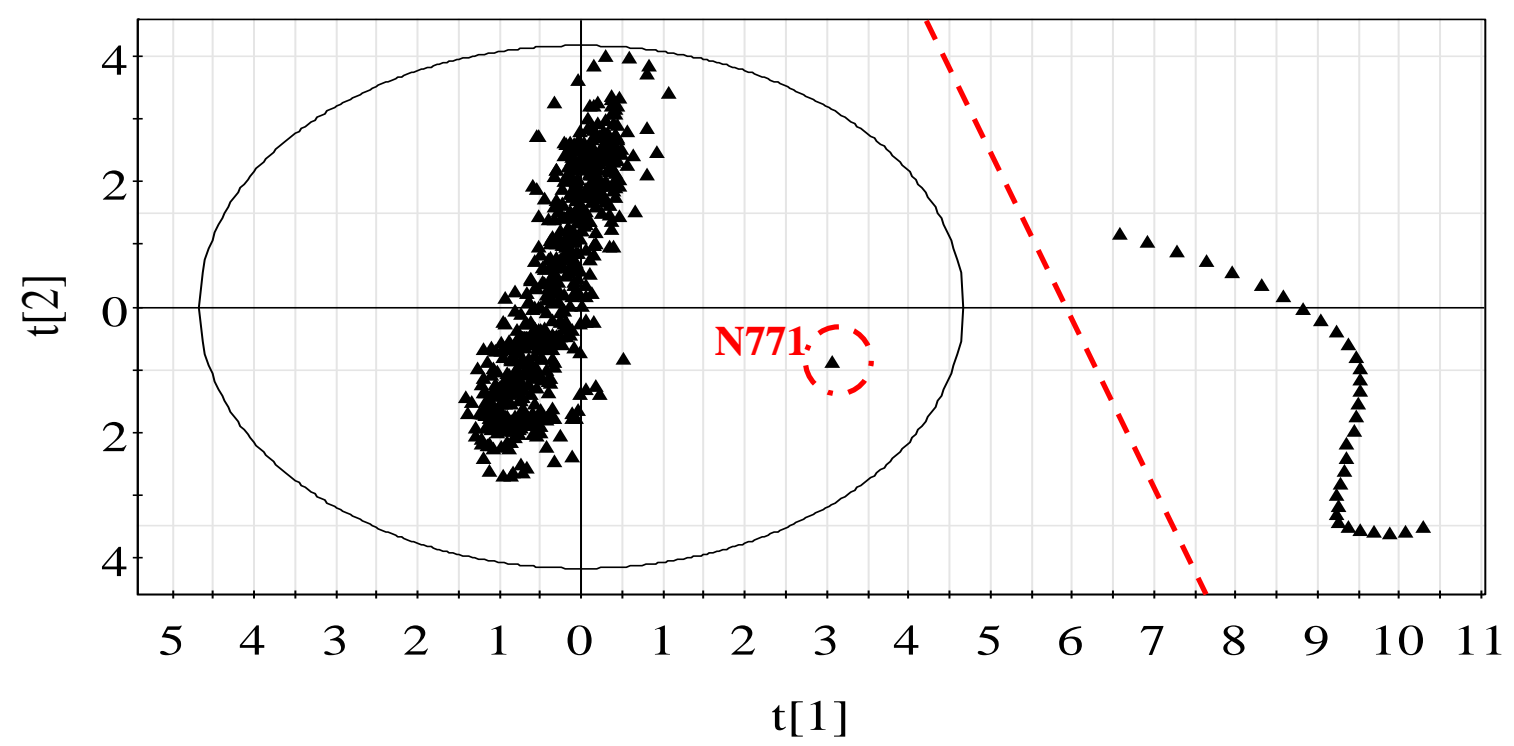




\section{PCA_Loading Plot_w*c[Comp. 1]/w*c[Comp. 2]}

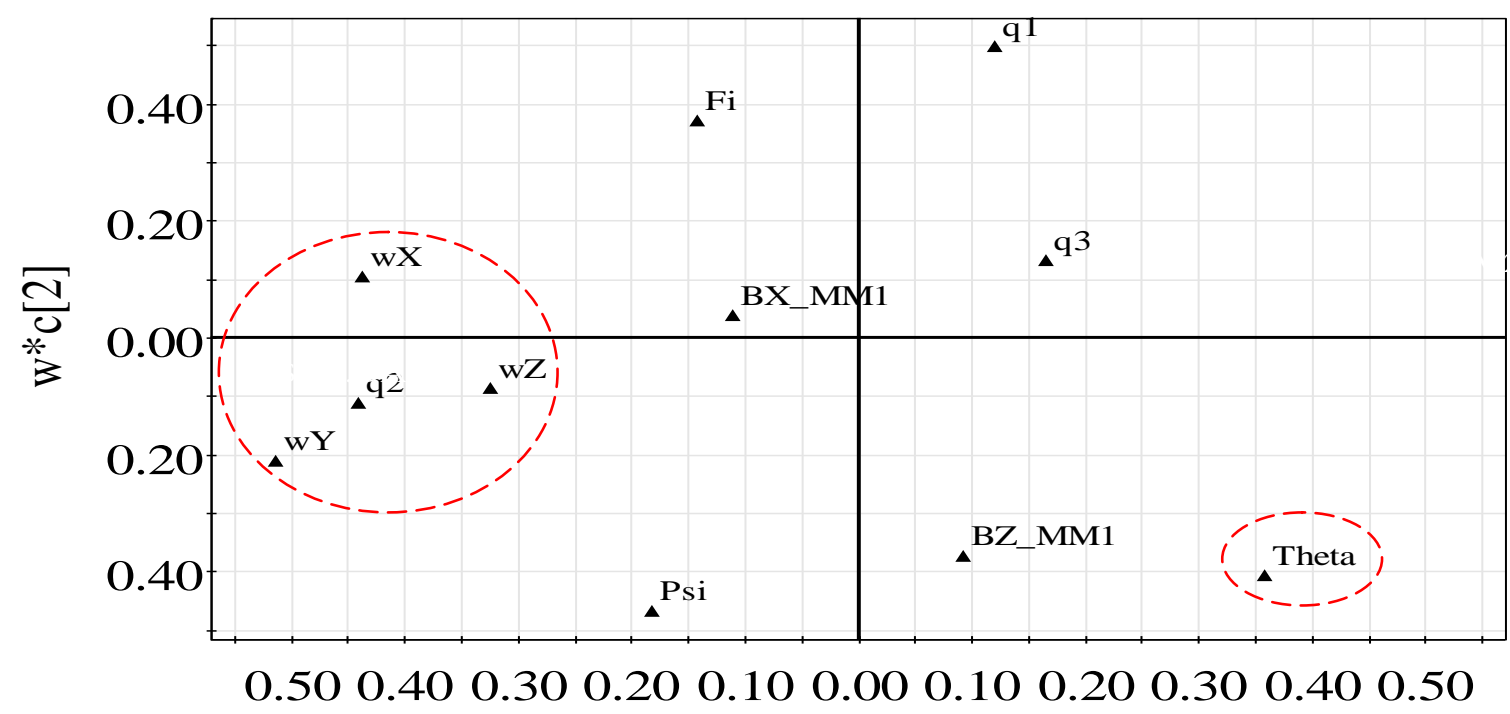

Fig. 9. SIMCA-P Score and Loading Plots using the Training Dataset

From fig. 8 and 9. one can notice, how angular velocities in $\mathrm{x}, \mathrm{y}$ and $\mathrm{z}$ directions $(\omega \mathrm{x}),(\omega \mathrm{y})$, $(\omega z)$ and quaternion (q2) in the left corner of the loading plot contribute to the left swarm (faulty states) of data in the score plot. In other words, these variables are directly correlated with the faulty states while the pitch angle (Theta) is inversely correlated with them. Further investigation carried out by using the contribution plot named variables importance to projection (VIP) bar plot fig. 10. The plot explains the anomaly identification across the features deemed most critical in determining overall health status of the process operation and shows that the aforementioned variables $\omega \mathrm{x}, \omega \mathrm{y}, \omega \mathrm{z}, \mathrm{q} 2$ and Theta are most critical.

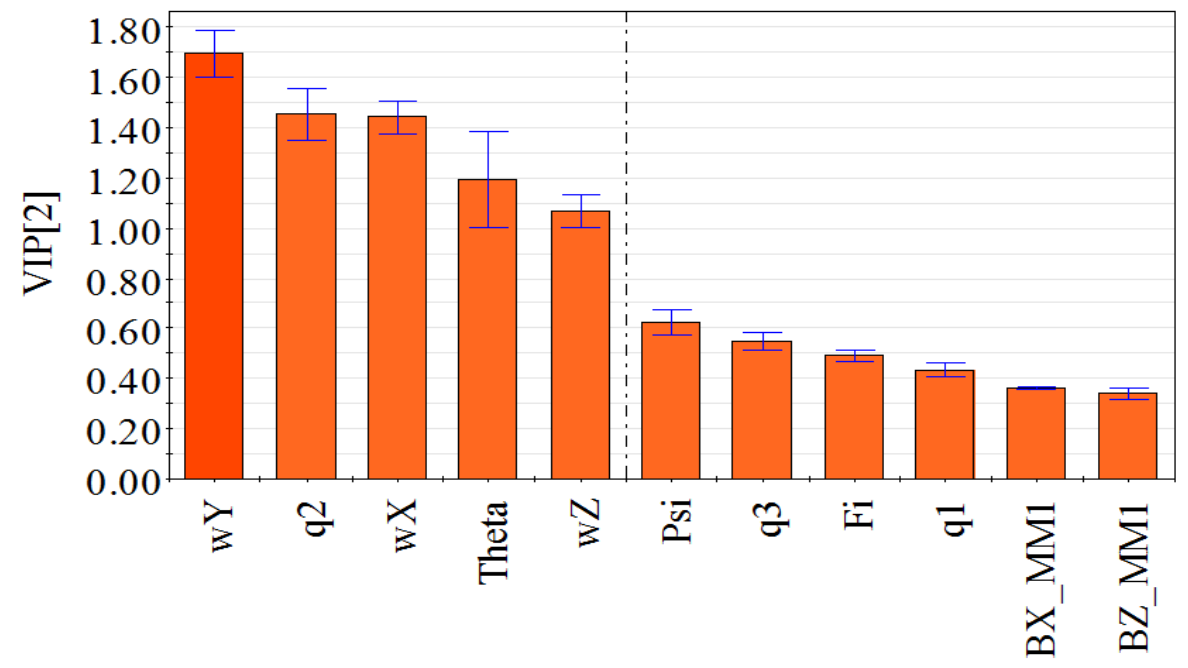

Fig. 10. SIMCA-P Variables Importance in Projection (VIP plot)

More investigation was carried out using contribution plots which represent the unexplained variation "residuals" in the model and can be used to define a control limit in a direction perpendicular to the PC-model hyperplane and detect processes upsets "spikes in telemetry data that couldn't be observed by scatter plots" by determining moderate outliers. Residual means the X-variation that was not captured by the PC-model, the variation which forms the matrix E.) By moderate outliers we mean outliers that not strong enough to shift the model 
plane. Fig. 11. and 12. demonstrate both our PCA contribution plot and the SIMCA-P plot that is named distance to model X (DModX). From both plots one can note that the most of the observations from "N1" to "N770" used to train the model are inside or nearly inside the model critical distance (D-Critical 0.05). This indicates that in this time interval the process is fairly stable and no new process event is recognized. However, the last observations from "N771" to "N810" don't fit the model well, and indicate that a new process behavior has become establish and ought to be scrutinized more closely and observation "N771" is farthest away from the model plane displays largest portion of unexplained variation.

PCA-X_Contribution Plot_DModX[Comp. 2]

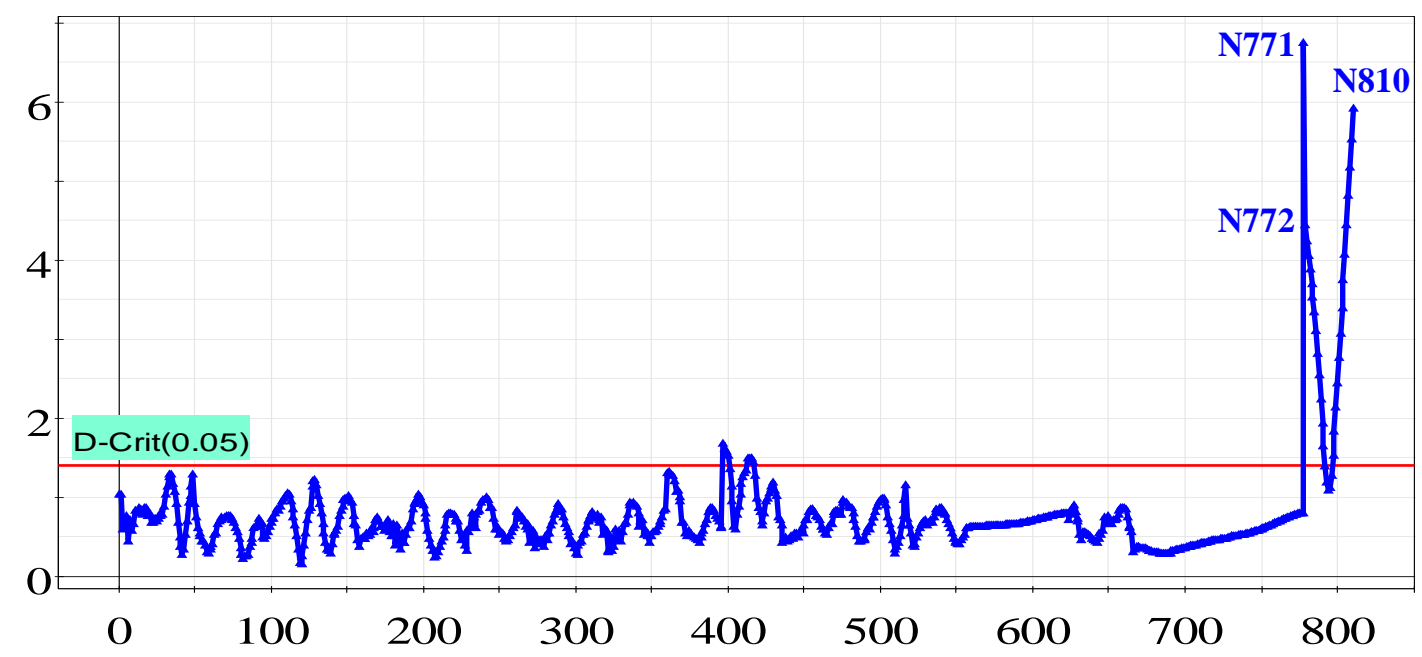

Fig. 11. PCA Algorithm Contribution Plots PCA Algorithm - Contribution Chart for Moderate Outliers

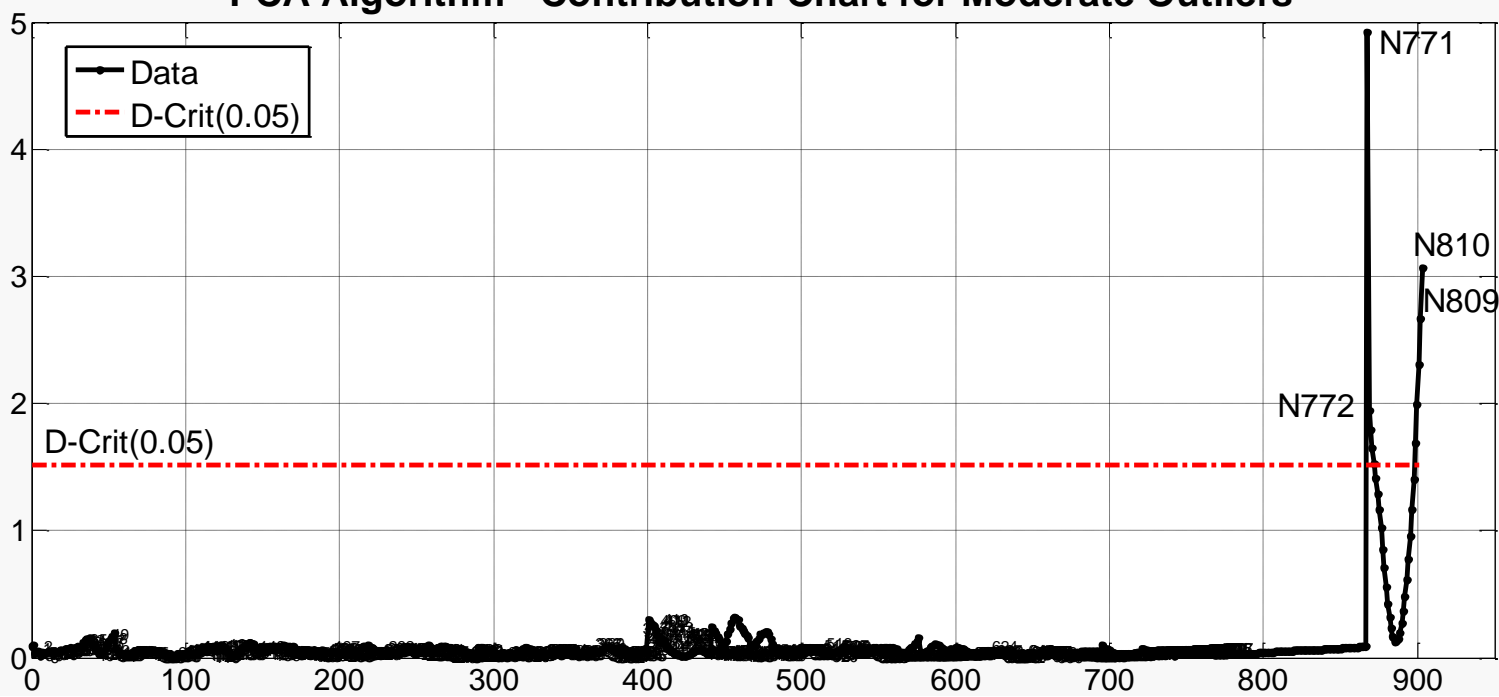

Fig. 12. SIMCA-P Contribution Plot Distance to Model X (DModX)

Further investigation can be carried out using control charts. Fig.13. shows the control Hotelling's $\mathrm{T}^{2}$ and DModX (model residual) plots with the training set. A clear advantage is realized from modeling the X-space over the present approaches the Hotelling's $\mathrm{T}^{2}$ chart failed to detect this shift but is alarmed through the model residual DModX starting from observation "N771". One can observe that the score plot model can't differentiate between the faulty telemetry "N771" with the others normal ones in both models results acquired by Simca-P and the proposed algorithm. The explanation for this is the difference in goal between supervised and unsupervised learning methods. The unsupervised PCA is a well- 
known multivariate technique for exploratory data analysis. But, supervised pattern recognition techniques differ from PCA in that the goal is to detect similarities between objects and find groups in the data on the basis of calculated distances, whereas PCA does not focus on how many groups will be found. Consequently, Also PCA do not use information related to predefined classes of objects. On the other hand, supervised pattern recognition requires a priori information on the set of samples that is used for classification purposes. Gathering both control and residual contribution charts are very informative and enables strong and moderate process outliers to be considered simultaneously.
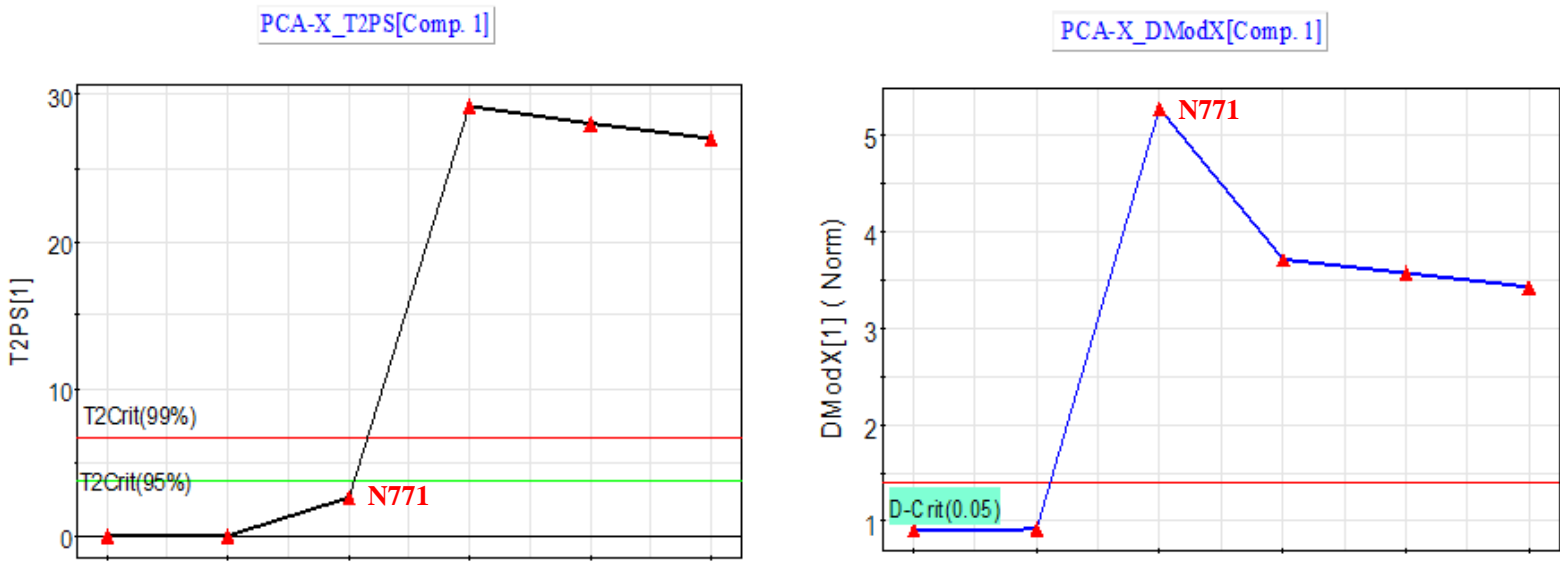

\section{Fig. 13. Monitoring System Built for Alarm}

Fig. 14. explores the contribution bar plot which is used to understand which variables contribute most strongly to an observed process change. One can notice that the angular velocity of spacecraft $\omega y$, quaternion elements $q 2$ of the satellite orientation in orbital coordinate system (OCS) and the pitch angle (Theta) giving an alarm on the occurrence of the faulty state due to high angler velocity which caused by the beginning of high rate damping mode (detumbling mode) occurs. Also the dissimilarity between faulty observation "N771" and the rest faulty ones is a critical intention. This unique direction is regarding to that faulty telemetry "N772" has the higher effect of the pitch angle (Theta) instead of reverse effect in others faulty telemetry and this is reasonable, due to the beginning of occurrence of high rate damping mode of the spacecraft.
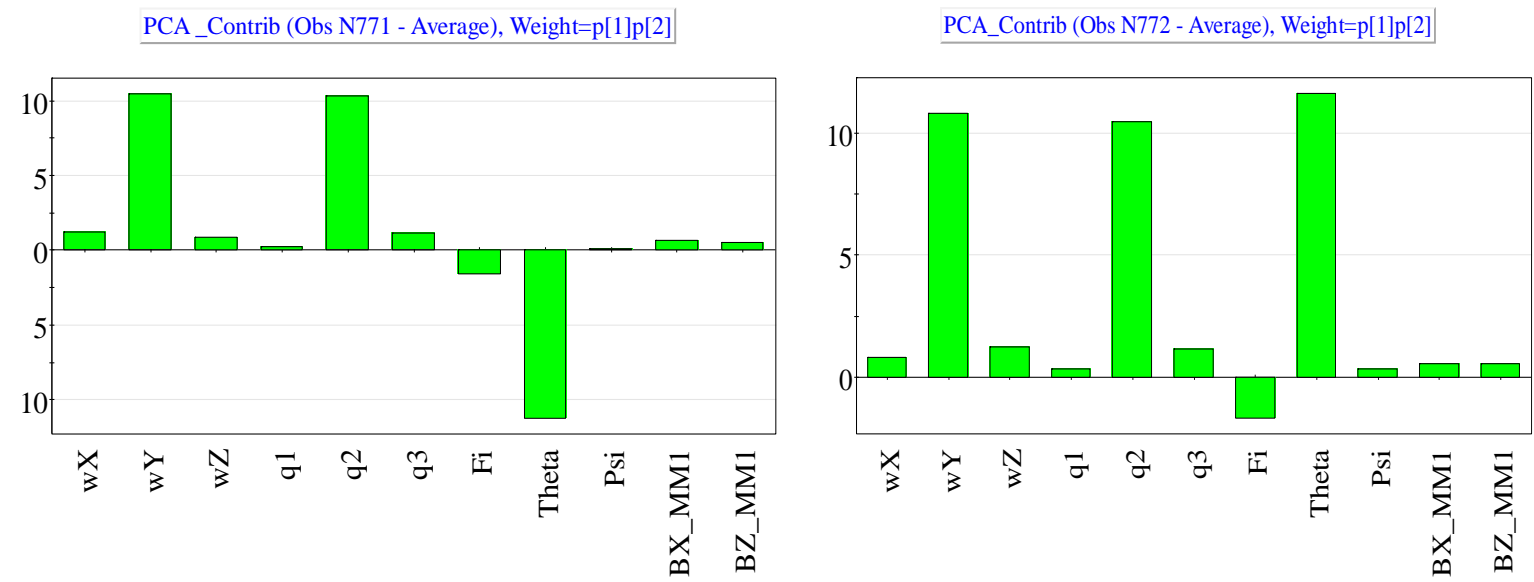

Fig. 14. Contribution to Variables Weight Plot

Additional analysis using multivariate control charts are crucial objective because the contribution plots will not explicitly indicate the cause of this shift provides good insight into 
plausible causes or variables related to the causes. In order to detect special events, control chart technique is routinely used to monitor key process variables and quality over time. So, the proposed algorithm has the capability to to detect large changes like a shift in average or general drift overtime via investigating the telemetry data over time. Fig. 15. And 16. show that the process shifts could be tackled using the control chart of the PCA algorithm and SIMCA-P "Shewhart control chart" which are used as an early warning trend monitor. Gathering both control and residual contribution charts are very informative and enables strong and moderate process outliers to be considered simultaneously.

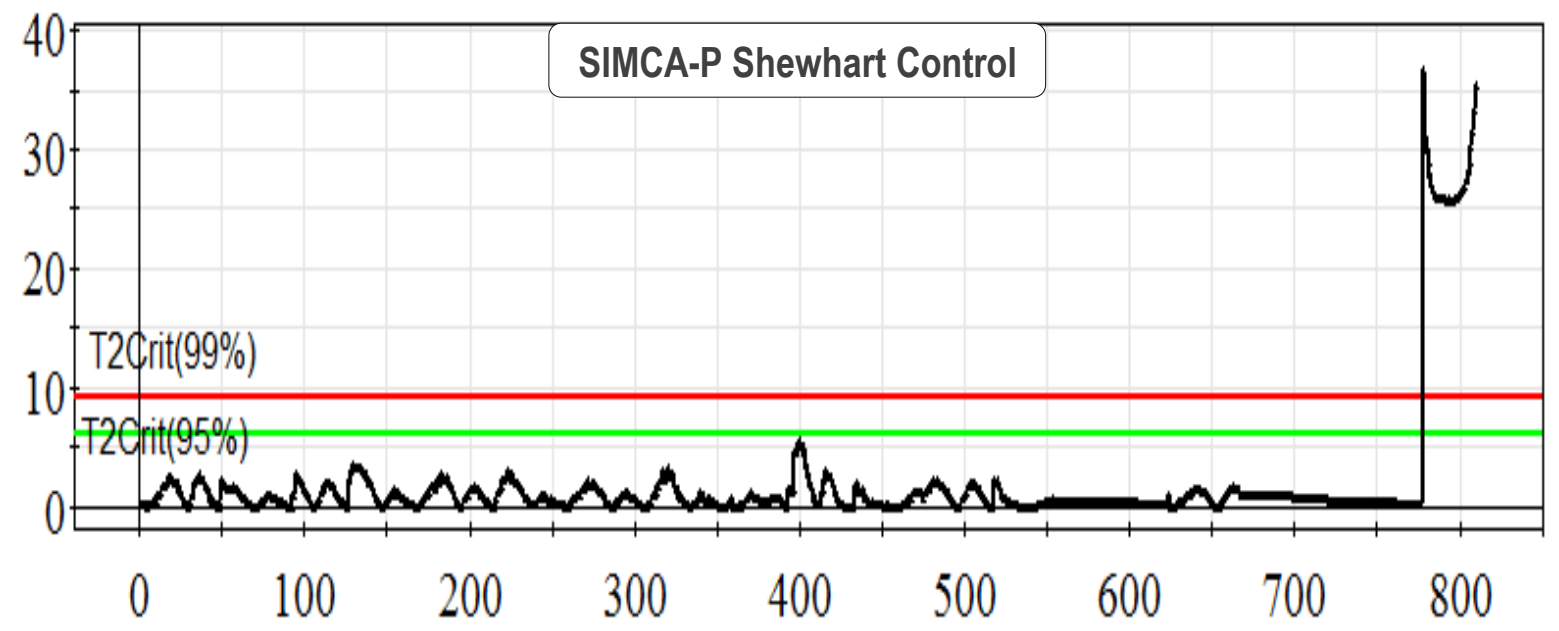

Fig. 15. SIMCA-P Shewhart Control Charts for Process Shifts PCA Algorithm- Control Chart for Process Shifts

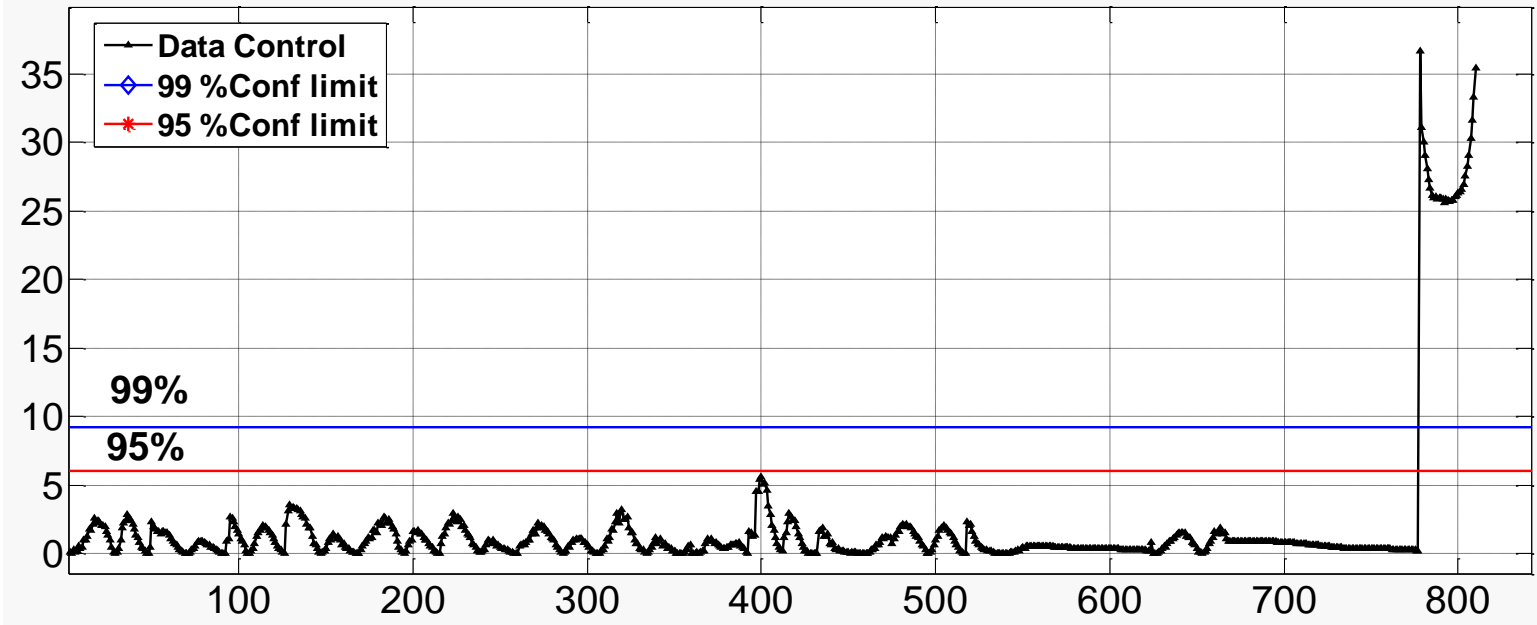

Fig. 16. PCA Algorithm Control Charts for Process Shifts

\section{Conclusions and Future Directions}

The principle component analysis (PCA) unsupervised statistical learning algorithm is introduced to monitor the spacecraft operations status via practical application on the attitude determination and control subsystem (ADCS). The algorithm is based on the multivariate projection technique and applied to spacecraft telemetry data in order to manage the ADCS operations and overcome faulty state. The algorithm exploits telemetry data for model building and testing. The analysis results show that the algorithm has the capability to supervise the major trends in the data and compares the characteristics of each one and capable to correlate the clusters with low cardinalities to their corresponding trigger events. Furthermore, the analysis results were compared with the SIMCA-P software and show an 
evident agreement. The algorithm proves that it can be used as an effective tool for monitoring the ADCS operations status and deals with multiple process states.

In the near future, we aim at adopting and evolving the approach into a more powerful tool by extending the work to be implemented for more than one faulty system and coupled with supervised learning techniques like support vector machines (SVMs) and projection to latent structure discriminant analysis (PLS-DA) to solve the relevant problems.

\section{References}

[1] Iverson, D.L., Martin, R., Schwabacher, M., Spirkovska, L., Taylor, W., Mackey,R., and J. Castle, J.P. "General Purpose Data-Driven System Monitoring for Space Operations," AIAAInfotech @ Aerospace Conference, Seattle, WA, October 2010.

[2] David L. Iverson "Data Mining Applications for Space Mission Operations System Health Monitoring," NASA Ames Research Center, Moffett Field, California, 94035 Space Operations Conference, 2008.

[3] Takehisa Yairi, Minoru Inui, Akihiro Yoshiki, Yoshinobu Kawahara, Noboru Takata, "Spacecraft Telemetry Data Monitoring by Dimensionality Reduction Techniques," SICE Annual Conference, 2010.

[4] Ivano Verzola, Anne-Emmanuelle Lagny and Janos Biswas " A Predictive Approach to Failure Estimation and Identification for Space Systems Operations," 13th international conference on space operations, Pasadena, California ,USA, May 2014.

[5] John MacGregora; Ali Cinarc; "Monitoring, fault diagnosis, fault-tolerant control and optimization: Data driven methods," Journal of Computers and Chemical Engineering 47, June 2012.

[6] Jian Peng, Linjun Fan,Weidong Xiao, and Jun Tang "Anomaly Monitoring Method for Key Components of Satellite," Scientific World Journal Volume 2014, Article ID 104052, January 2014.

[7] Steve Lindsay and Diane Myung-kyung Woodbridge "Spacecraft State-of-health (SOH) Analysis via Data Mining," $13^{\text {th }}$ international conference on space operations, Pasadena, California ,USA, May 2014.

[8] Wold S., Esbensen K. and Geladi P., "Principal component analysis", Chemometrics and intelligent laboratory systems, 2: 37-52, 1987.

[9] Jackson J.E., A User's Guide to Principal Components, Wiley, New York, 1991.

[10] Wold S., Sjostrom M. and Eriksson L., "PLS-regression: a basic tool of Chemometrics", Chemometrics and intelligent laboratory systems, 58:109-130, 2001.

[11] L. Simar and W.Hardle, "Applied Multivariate Statistical Analysis," Tech method and data technologies, Springer Verlag, Berlin and Louvain-la-Neuve, 2003.

[12] P.R.Goulding, B.Lennox, D.J.Sandoz, K. J.Smith and Marjanovic, "Fault detection in continuous processes using multivariate statistical methods," International journal of systems science, 31(11), pp. 1459-1471, 2000.

[13] T.Kourti Patricia Ralston, Gail DePuy, and James H. Graham, "Computer-based monitoring and fault diagnosis a chemical process case study," Instrument Society of America Transactions ISA, Vol. 40, , pp. 85-98. E, USA, 2003.

[14] Yimin Zhan and Viliam Makis, "A robust diagnostic model for gearboxes subject to vibration monitoring," Journal of Sound and Vibration 290, pp. 928-955, 2006.

[15] Hotelling, H."Analysis of a complex of statistical variables into principal components," Journal of Educational Psychology, 24, 417- 441, 1933.

Qin, S. "Statistical process monitoring: basics and beyond," Journal of Chemometrics, 17(8/9), 480-502, 2003. 